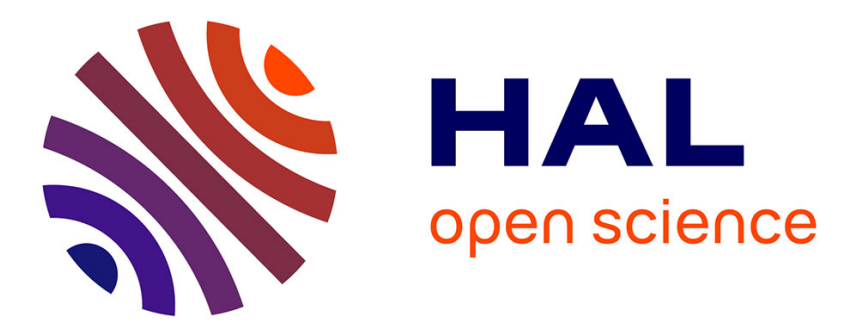

\title{
Calibration and uncertainty estimation of non-contact coordinate measurement systems based on Kriging models
}

\author{
Lan Fei, Jean-Yves Dantan, Cyrille Baudouin, Shichang Du
}

\section{To cite this version:}

Lan Fei, Jean-Yves Dantan, Cyrille Baudouin, Shichang Du. Calibration and uncertainty estimation of non-contact coordinate measurement systems based on Kriging models. Precision Engineering, 2019, 57, pp.16 - 29. 10.1016/j.precisioneng.2019.02.004 . hal-03484729

\author{
HAL Id: hal-03484729 \\ https://hal.science/hal-03484729
}

Submitted on 20 Dec 2021

HAL is a multi-disciplinary open access archive for the deposit and dissemination of scientific research documents, whether they are published or not. The documents may come from teaching and research institutions in France or abroad, or from public or private research centers.
L'archive ouverte pluridisciplinaire HAL, est destinée au dépôt et à la diffusion de documents scientifiques de niveau recherche, publiés ou non, émanant des établissements d'enseignement et de recherche français ou étrangers, des laboratoires publics ou privés.

\section{(ㄷ)(1) $\$$}

Distributed under a Creative Commons Attribution - NonCommerciall 4.0 International 


\title{
Calibration and uncertainty estimation of non-contact coordinate measurement systems based on Kriging models
}

Lan Fei, Jean-Yves Dantan, Cyrille Baudouin, Shichang Du

\begin{abstract}
Non-contact three dimensional (3D) coordinate measurement systems (CMSs) using optical scanning techniques have the advantage of fast acquiring large numbers of points. However, these systems are known to be less accurate in comparison to the contact-based counterparts. To improve the measurement accuracy of non-contact 3D CMSs, a novel Kriging models-based calibration and uncertainly estimation method is proposed. The spatial correlation of measurement uncertainty is investigated and the calibration values for unmeasured points based on Kriging models are estimated. A procedure of best model selection is presented, and the influence of the calibration model parameters is analyzed. The proposed calibration method is validated through an ATOS II triple scan system. The results show that a significant accurate improvement for the non-contact $3 \mathrm{D}$ measurement system is achieved.
\end{abstract}

\section{Keywords:}

coordinate measurement system;

non-contact measurement;

measurement uncertainty;

calibration;

Kriging model 


\section{Introduction}

Since three dimensional (3D) coordinate measurement systems (CMSs) with optical imaging devices offer the option to output high density data for parts at high speed, they have been widely used in lab and industry [1-4]. In recent years, non-contact optical measurement systems have not only been seen with significant advances in technical developments, but also undergone a boost in applications, such as surface quality improvement [5-8], surface error evaluation [9], surface separation [10, 11], and tool wear monitoring [12]. However, for actual camera lenses, such as a fixed length lens, a zoom lens or even an expensive high-quality telecentric lens, image distortions unavoidably exist due to lens aberrations, misalignment of optical elements and non-parallelism between image plane and sensor plane [13-15]. Due to the non-uniform characteristics of camera lens distortion, actually the coordinates on object surface may cause different values in the sensor plane. Therefore, measurement results are usually affected by measurement uncertainty, which could lead to technical and economic risks in industrial applications.

Over the last 30 years, two different methods have been adopted to achieve high accuracy, namely, uncertainty avoidance and calibration. Uncertainty avoidance is the traditional method focusing on the reduction of possible uncertainty sources in the design and manufacturing processes [16]. Careful design and precise construction can reduce the uncertainty, but every subsequent micrometer/nanometer of uncertainty reduction will cause an exponentially increasing cost. So it is practical to calibrate CMSs between the measurement coordinate and the actual object.

The calibration and uncertainty estimation method has been explored as a cost-effective method of improving the accuracy of CMSs [17-20]. Early developments in calibration are well described by Evans [21]. Some effective methods are reported in the literature to model and calibrate CMSs. These methods include polynomial models and autoregressive moving average models [22], homogeneous coordinate transformation (HTM) methods [23], neural network based methods [24-28], D-H models [29, 30], fuzzy error interpolation techniques [31], iterative learning and decoupling methods [32, 33], recursive least squares identification 
techniques [34], Monte Carlo method [35] and other analytical methods [36-41].

So far, limited research on the spatial statistics-based methods to model and calibrate CMSs considering the spatial correlation of the measurement data has been conducted. The spatial correlation arises frequently in data measured within certain intervals of space, and the data indeed exhibits a significant amount of positive autocorrelation [42]. Measurements are often spatially correlated because they are obtained in similar manufacturing conditions and related to similar properties of the machined material [43]. Spatial correlation is different from temporal correlation, which is usually represented via time series models. In fact, spatial correlation models allow one to represent contiguity in space rather than in time. Spatial statistics, e.g. Kriging method, is one of most important meta-models for describe spatial correlations in random field [44]. Detailed descriptions of existing research on Kriging meta-models are provided in a review [45].

The literature on spatial statistics-based calibration methods is sparse. A method based on Co-Kriging models to estimate the errors of the surface form not only concerning spatial correlation but also concerning the influence of machining conditions is developed in [46]. A spatial statistical method to design adaptive inspection plans for the geometric control of mechanical parts with CMSs is presented in [47]. The prediction uncertainty of geometric deviations is provided using Kriging models. A Kriging-based procedure to identify the minimum of measured points to check the conformity with a given confidence level in the inspection of large surfaces is presented in [48]. An on-line inspection system with error calibration to obtain high machining accuracy for free-form surface components is proposed in [49]. Through the spatial statistical analysis of the residual errors of a regression model, the errors are decomposed into systematic errors and random errors. The spatial error compensation methods for computer numerical control (CNC) machining center is proposed in $[50,51]$.

In these last applications, Kriging-based procedure to improve inspection has proved to be effective. This paper develops a Kriging-based method to achieve the calibration and uncertainty estimation of non-contact 3D CMSs. The advantages of this proposed solution are 
many in comparison to the classical approaches:

- It is not necessary to develop a complex phenomenological model of the sensor. The systematic error of a simplified model of the sensor was corrected by the Kriging model.

- It is possible to adopt an adaptive approach for the calibration. Based on the uncertainty estimation of the kriging model, it is possible to identify the spatial area that should be recalibrated.

The proposed Kriging-based method for the calibration of non-contact 3D CMSs is presented in Section 2. An application of the approach is illustrated through a case study in Section 3.

\section{The proposed calibration method}

The aim of this proposed calibration method is to correct the systematic error of the simplified model of the sensor by a kriging model. To do so, a gauge is measured. It is important to know the geometrical characteristics of this gauge. In this application, the GOM gauges are used.

\subsection{The calibration procedure}

Fig. 1 shows the calibration procedure of a measurement system, and the main steps are described as follows.

\section{[Insert Fig. 1 here]}

Step 1. Obtaining measured points and theoretical reference points of the gauge. A measured point $S$ obtained by 3D CMS is denoted as,

$$
s_{i \text {-measured }}=\left(x_{i-\text { measured }}, y_{i-\text { measured }}, z_{i \text {-measured }}\right)
$$

where $x_{i \text {-measured }}, y_{i \text {-measured }}, z_{i-\text { measured }}$ are three dimensional coordinates of a measured point respectively. These values are obtained from the simplified model of the sensor.

Based on the geometrical characteristics of the gauge, the coordinate values of theoretical reference points are determined by an orthogonal projection of the measured 
points on the gauge surface. Each measured point is mapped into the corresponding theoretical reference point,

$$
s_{i \text {-theoretic }}=\left(x_{i \text {-theoretic }}, y_{i-\text { theoretic }}, z_{i \text {-theoretic }}\right)
$$

where $x_{i \text {-theoretic }}, y_{i-\text { theoretic }}, z_{i-\text { theoretic }}$ are three dimensional coordinates of the theoretical reference points respectively.

Illustration: Case of the sphere (Figure 1) - To define the theoretical reference points, the gauge sphere is fitted to the measured points and each measured point is projected on the fitted gauge sphere.

Step 2. Calculating the measurement errors. A measurement error is the distance between the measured point and theoretical reference point at each direction of the axis X, Y, and Z,

$$
\Delta s_{i}=\left(\Delta x_{i}, \Delta y_{i}, \Delta z_{i}\right)=\left(x_{i-\text { measured }}, y_{i \text {-measured }}, z_{i-\text { measured }}\right)-\left(x_{i-\text { theoretic }}, y_{i-\text { theoretic }}, z_{i \text {-theoretic }}\right)
$$

A measurement error is discrete in each measured point on the surface, which needs to be calibrated. It is necessary to use spatial interpolation method to estimate the whole surface of the new measured part surface to realize the calibration of 3D measurement systems. The measurement error estimation model is established and applied at any point of the whole standardized part surface.

Step 3. A Kriging model is used to estimate the calibration $\Delta C_{0}=\left(\Delta x_{0}, \Delta y_{0}, \Delta z_{0}\right)$ on the specified untried site $s_{0}$ by the response. The measurement errors $\Delta C_{i}=\left(\Delta x_{i}, \Delta y_{i}, \Delta z_{i}\right)$ at a set of measured points $s_{i \text {-measured }}=\left(x_{i \text {-measured }}, y_{i \text {-measured }}, z_{i-\text { measured }}\right)$ (see Fig. 2) are represented as,

$$
\begin{aligned}
& \Delta x_{0}=u\left(x_{i-\text { measured }}, y_{i-\text { measured }}, z_{i-\text { measured }}\right)=u\left(s_{i \text {-measured }}\right) \\
& \Delta y_{0}=v\left(x_{i-\text { measured }}, y_{i-\text { measured }}, z_{i-\text { measured }}\right)=v\left(s_{i \text {-measured }}\right) \\
& \Delta z_{0}=w\left(x_{i-\text { measured }}, y_{i-\text { measured }}, z_{i-\text { measured }}\right)=w\left(s_{i-\text { measured }}\right)
\end{aligned}
$$

where $u, v, w$ are the Kriging models with different regression models and correlation models respectively. 


\section{[Insert Fig. 2 here]}

Step 4. The calibration of any areas can be estimated by the Kriging spatial interpolation model. Therefore, this estimation could be used to calibrate the measurement error for a new measurement, which realizes the goal of calibration of 3D CMSs.

\subsection{Kriging-based calibration model}

Kriging is one of most important spatial statistics-based stochastic process prediction methods used to produce contour maps of surfaces derived from regularly or irregularly scattered points in a space. Let $D$ be the region of a standardized part surface where the calibration value of measurement error $\Delta C$ is predicted. And $n$ points $s=\left(s_{1}, s_{2}, \cdots, s_{n}\right)$ are measured with $s_{i} \in D$ for $i=1,2, \cdots, n$. The measurement error $\Delta C$ (see Fig. 3) can be represented as,

$$
\Delta C(s)=\mu(s)+\delta(s), s \in D
$$

where $s$ is the variable $s=\left[s_{1}, s_{2}, \cdots, s_{n}\right]^{T}, \Delta C(\cdot)$ is regionalized measurement error, $\mu(\cdot)$ is the expectation of deterministic structure and $\delta(\cdot)$ is a stationary random function with zero mean and known dependence structure.

\section{[Insert Fig. 3 here]}

In order to develop the calibration model, the form of the deterministic structure $\mu(\cdot)$ can be specified by three types of Kriging models:

- The simple Kriging: $\mu(s)=$ constant . The constant is known.

- The ordinary Kriging: $\mu(s)=\beta_{0}$. The constant is unknown and estimated from the data.

- The universal Kriging: $\mu(s)=\sum_{j=1}^{p} f_{j}(s) \beta_{j}$. It is a polynomial function of order 1 (linear universal Kriging, LUK) and of order 2 (quadratic universal Kriging, QUK) 
at the location $s$ with three dimension $\mathrm{x}, \mathrm{y}$ and $\mathrm{z}$. And ordinary Kriging can be considered as a special universal Kriging with $p=1$ and $f_{1}(s)=1$.

The measurement error $\Delta C(s)$ for $s \in D$ is considered as a realization of a Gaussian random process:

$$
\Delta C(s)=\beta^{\prime} f(s)+\delta(s)
$$

where $f(s)=\left(f_{1}(s), f_{2}(s), \cdots, f_{p}(s)\right)^{\prime}$ is a set of specified trend functions and $\boldsymbol{\beta}=\left(\boldsymbol{\beta}_{1}, \boldsymbol{\beta}_{2}, \cdots, \boldsymbol{\beta}_{p}\right)^{\prime}$ is a set of coefficients.

The measurement error $\Delta C(s)$ satisfies a Gaussian random process with zero mean and stationary covariance.

$$
\begin{aligned}
& \mathrm{E}(\Delta C(s))=\beta^{\prime} f(s) \\
& \operatorname{Cov}(\Delta C(s), \Delta C(s+d))=\sigma_{\delta}^{2} R(d, \theta)
\end{aligned}
$$

where $\sigma_{\delta}^{2}$ is the process variance, and $R$ is the stationary correlation function (SCF).

The SCF depends only on the displacement vector $\boldsymbol{d}$ between any pair of points in $D$ and on a set of hyper-parameters $\theta$.

$$
R\left(\theta, s_{i}, s_{j}\right)=\prod_{i, j=1}^{n} R_{j}\left(\theta, s_{i}-s_{j}\right)
$$

Base on equation (2) and (3), the joint random variable, measurement error, $\left(\Delta \mathrm{C}\left(\boldsymbol{s}_{0}\right), \Delta \mathrm{C}\left(\boldsymbol{s}_{1}\right), \Delta \mathrm{C}\left(\boldsymbol{s}_{2}\right), \cdots, \Delta \mathrm{C}\left(\boldsymbol{s}_{n}\right)\right)$ satisfies a multivariate normal function, $N\left(\left(f_{0}^{\prime}, F\right)^{\prime} \beta, \sigma_{\delta}^{2} \Sigma\right)$ with

$$
\Sigma=\left(\begin{array}{ll}
1 & r_{0}^{\prime} \\
r_{0} & R
\end{array}\right)
$$

where $r_{0}$ is the correlation vector $r\left(s_{0}\right)=\left[R\left(\theta, s_{1}, s_{0}\right) \cdots R\left(\theta, s_{m}, s_{0}\right)\right]^{T}$, and $R$ is the $n \times n$ correlation matrix whose $(i, j)$ element is $R\left(d_{i j}=s_{i}-s_{j}\right)$.

According to the best unbiased estimate condition of Kriging method, the mean of estimation of the calibration value $\Delta C(s)$ at the point $s_{0}$ is: 


$$
\mu_{\Delta \hat{C}}\left(s_{0}\right)=\Delta \hat{C}\left(s_{0}\right)=f\left(s_{0}\right) \cdot \beta^{*}+r\left(s_{0}\right) \cdot \gamma^{*}=f_{0}^{\prime} \beta^{*}+r_{0}^{\prime} R^{-1}\left(\Delta C^{n}-F \beta^{*}\right)
$$

where $f_{0}$ is the $p \times 1$ vector of the trend functions in $s_{0}, F$ is the $n \times p$ matrix of $\left\{f_{j}\left(s_{i}\right)\right\}_{i=1, \cdots, n, j=1, \cdots, p}$ of the trend functions calculated in $\left(\boldsymbol{s}_{1}, \boldsymbol{s}_{2}, \cdots, \boldsymbol{s}_{n}\right), \beta$ is unknown coefficient, and it is generalized by LS estimator $\hat{\beta}^{*}=\left(F^{\prime} R^{-1} F\right)^{-1} F^{\prime} R^{-1} \Delta C$.

Moreover, a confidence index about the prediction, called the Kriging variance, is the mean squared error between the prediction $\Delta \hat{C}(s)$ and the actual value $\Delta C(s)$. It is given by:

$$
\sigma_{\Delta \hat{C}}^{2}\left(s_{0}\right)=E\left(\left(\Delta \hat{C}_{0}-C\left(s_{0}\right)\right)^{2}\right)=\sigma_{\delta}^{2}\left(1-r_{0}^{\prime} R^{-1} r_{0}+g_{0}^{\prime}\left(F^{\prime} R^{-1} F\right)^{-1} g_{0}\right)
$$

where $\quad g_{0}=f_{0}-F^{\prime} R^{-1} r_{0} \quad, \quad f_{0}: f\left(\boldsymbol{s}_{0}\right), \quad r_{0}: r\left(\boldsymbol{s}_{0}\right) ; \quad F:\left[f\left(\boldsymbol{s}_{1}\right) \cdots f\left(\boldsymbol{s}_{n}\right)\right], \quad R:\left[r\left(\boldsymbol{s}, \boldsymbol{s}_{1}\right) \cdots r\left(\boldsymbol{s}, \boldsymbol{s}_{n}\right)\right]$ and $\sigma_{\delta}^{2}$ is the variance of the process.

$$
\sigma_{\delta}^{2}=\frac{1}{m}\left(\Delta C-F \beta^{*}\right)^{\prime}\left(\Delta C-F \beta^{*}\right)
$$

Kriging is an exact interpolation method, so predictions made at any point $s$ give $\Delta \hat{C}(s)=\Delta C(s)$. Thus, the Kriging variance is null at these points.

\subsection{Cross-validation of calibration model}

Cross-validation is a model validation technique for assessing how the results of a statistical analysis generalize to an independent data set. It is mainly used to estimate how accurately a predictive model performs in practice. In order to better realize the calibration of a measurement system, the cross-validation technique is applied to select the best regression model and correlation model.

Fig. 4 shows the procedure of cross-validation. Several points of the measurement errors (testing data) are temporarily eliminated and then the calibration values of the measurement systems are estimated by Kriging methods using the remaining data (training data). This operation is repeated for some or all points. Thus at any observation point, it is calculated by Kriging with an estimated value $\Delta C^{*}$ and an estimation variance of Kriging $\sigma_{\delta}^{2}$. 


\section{[Insert Fig. 4 here]}

The main steps are described as follows.

Step 1: Generate measurement error with three dimensions $\Delta C_{i}=\left(\Delta x_{i}, \Delta y_{i}, \Delta z_{i}\right)$ by the $\mathrm{N}$ measured points and nominal points which are projected on the fitting surface obtained by LS method.

Step 2: Select $M$ points as measured points for the estimation of calibration and temporarily eliminate the measurement error of the remaining (N-M) points.

Step 3: Calculate the estimation of calibration and Kriging variance associated in the remaining $(\mathrm{N}-\mathrm{M})$ points.

Step 4: Repeat $q$ times by steps (2) to (3) for each of M.

Step 5: Calculate the cross-validation criterion.

Step 6: Build the box plot (box-whisker plot) for evaluation value of cross-validation criterion on the M points.

Once the cross-validation is performed, several important criterions are used to evaluate the performances of the different Kriging methods. Here is a list of the most popular cross-validation criterions.

mean error: $M E=\frac{1}{n} \sum_{i=1}^{n}\left[\Delta C\left(s_{i}\right)-\Delta C^{*}\left(s_{i}\right)\right]$

mean squared error: $M S E=\frac{1}{n} \sum_{i=1}^{n}\left[\Delta C\left(s_{i}\right)-\Delta C^{*}\left(s_{i}\right)\right]^{2}$

root mean squared error: $R M S E=\sqrt{\frac{1}{n} \sum_{i=1}^{n}\left[\Delta C\left(s_{i}\right)-\Delta C^{*}\left(s_{i}\right)\right]^{2}}$

average Kriging standard error: $A K S E=\sqrt{\frac{1}{n} \sum_{i=1}^{n} \sigma_{\delta}^{2}\left(s_{i}\right)}$

mean standardized prediction error : $M S P E=\frac{1}{n} \sum_{i=1}^{n} \frac{\Delta C\left(s_{i}\right)-\Delta C^{*}\left(s_{i}\right)}{\sigma_{\delta}\left(s_{i}\right)}$

root mean square standardized prediction error: $R M S P E=\sqrt{\frac{1}{n} \sum_{i=1}^{n}\left[\frac{\Delta C\left(s_{i}\right)-\Delta C^{*}\left(s_{i}\right)}{\sigma_{\delta}\left(s_{i}\right)}\right]^{2}}$ 
where $\Delta C\left(s_{i}\right)$ is the real measurement error in the site, $\Delta C^{*}\left(s_{i}\right)$ is estimated calibration in the site, $\sigma_{\delta}\left(s_{i}\right)$ is the Kriging standard variance value and $n$ is the number of measured points used for the Kriging estimation.

The model is chosen if the cross-validation criterions satisfy:

1) Mean estimation errors (ME) and mean standardized prediction error (MSPE) are close to 0 . This criterion ensures the absence of bias.

2) The variance of the estimation errors (MSE, RMSE) is lower. This criterion reflects the robustness of the estimation and it provides the information on the estimation accuracy.

3) The variance of the standard errors (RMSPE) is closer to 1. It indicates that the standard deviation of Kriging properly reflects the estimation accuracy.

\section{Applications}

In this section, several experiments were conducted to illustrate how the proposed method based on Kriging calibrates a 3D measurement system and realize the measurement more precise. The calibration evaluation is analyzed with the comparison for ordinary Kriging and universal Kriging.

\subsection{Experimental setup}

The stereoscopic sensor (ATOS II Triple Scan, GOM) is installed on the manipulator (Fig. 5), which can be used to scan complex surfaces. The measurement system GOM is calibrated through the proposed calibration method based on a simplified phenomenological model of the sensor.

\section{[Insert Fig. 5 here]}

The experiment measurements were executed on the planes of gauge (see Fig. 6) and spheres of the gauge (see Fig. 7). To eliminate the influence of plane position, different positions of planes were measured by the sensor GOM: lower plane, middle plane, upper plane (blue), and diagonal plane (green) shown in Fig. 6. 


\section{[Insert Fig. 6 here]}

\section{[Insert Fig. 7 here]}

\subsection{Calculate measurement uncertainty}

In order to reduce systematic errors and realize calibration of the GOM measurement system, it is firstly important to calculate measurement error on the surfaces.

\subsubsection{Plane case}

To model the calibration of the GOM sensor on the datum plane, $\Delta \mathrm{z}$, or $\Delta \mathrm{z}$ and $\Delta \mathrm{y}$ are represented as the measurement error corresponding to the different positions of the plane. For lower, middle, and upper planes inclined with small angle (less than $10^{\circ}$ ), $\Delta \mathrm{z}$ is used to represent the calibrations, and for the diagonal plane inclined with large angle (around $45^{\circ}$ ), $\Delta \mathrm{z}$ and $\Delta \mathrm{y}$ are all used to represent the calibrations (see Fig. 8).

\section{[Insert Fig. 8 here]}

In Fig. 8, large dots are known as the measure, and the gauge plane is represented by the line. For the inclined plane with small angle, $\Delta \mathrm{z}$ are the differences between the measured points and projection points on the gauge plane. For inclined plane with large angle, $\Delta \mathrm{z}$ and $\Delta \mathrm{y}$ are the differences between the measured points (black) and projection points on the gauge plane.

The measured points in three dimensions $s_{i \text {-measured }}=\left(x_{i \text {-measured }}, y_{i \text {-measured }}, z_{i-\text { measured }}\right)$ were obtained by GOM sensor on the four planes. The gauge planes are shown in Table 1 .

\section{[Insert Tab. 1 here]}

According to equation (9) and Fig. 8, the measurement errors $\left(\Delta x_{i}, \Delta y_{i}, \Delta z_{i}\right)$ are calculated by the difference between the measured coordination data and the coordination of 
the projected points on the gauge plane. For lower, middle and upper planes, $\Delta z_{i}$ are calculated, while for diagonal plane, $\Delta y_{i}$ and $\Delta z_{i}$ are calculated as the measurement errors. The results of measurement errors on different planes are shown in Fig. 9.

\section{[Insert Fig. 9 here]}

\subsubsection{Sphere case}

The calibrated radius of the gauge sphere is $15.0004 \mathrm{~mm}$, and the coordinate of the sphere center is fitted by LS method using the measured points (see Fig. 10). The theoretical reference sphere (spherical datum surface curve) can be obtained. The measurement errors are the differences between the coordinates of actual measured points and the coordinates of their projection points on the spherical datum surface, expressed by $\left(\Delta x_{i}, \Delta y_{i}, \Delta z_{i}\right)$. The gauge sphere is shown in Table 2. The results of measurement errors on spherical surfaces are shown in Fig. 11. The measurement uncertainty of the calibrated radius of the gauge sphere has an impact on the errors assessment and therefore on the kriging model. GOM gauge was used in this application. The accuracy of the radius form is $0.0012 \mathrm{~mm}$ (GOM calibration certificate).

\section{[Insert Fig. 10 here]}

\section{[Insert Tab. 2 here]}

\section{[Insert Fig. 11 here]}

\subsection{Calibration results and discussion}

\subsubsection{Plane case}

According to the proposed calibration method based on Kriging for GOM sensor, the measurement errors calculated at different sites on the plane are considered as regional variables, and are used to estimate the calibrations in any other sites. For the lower plane, 1000 three dimensional data and the corresponding measurement errors $\Delta z_{i}$ are randomly sampled from 33678 measured points. Two experiments were conducted to provide 
calibrations in various areas: on the gauge plane and in a larger cubic space around the gauge plane. The measured points are collected and used to estimate the calibrations in Fig. 12.

\section{[Insert Fig. 12 here]}

The process of calibration calculation is explained for the unmeasured points in Fig. 12. For sampling in a larger cubic space, 1,000 points are collected by rectangular grid method in the larger cubic space around the fitting mean plane (Fig. 12 (a)). Then the calibrations of these 1000 points are predicted using previously calculated measurement error data $\Delta z_{i}$ selected from 33678 by random sampling method. While sampling on the fitting plane, 2912 (52* 56) points are collected by mesh grid method on the fitting mean plane (Fig. 12 (b)). Then the calibrations of these 2912 points are estimated using the same known measurement error data.

To estimate calibrations for sampling on the fitting plane, two methods are defined in the calculation (see Fig. 13). The first one is that the calibration is directly based on the positions of points $\Delta z_{0}=w\left(x_{i-\text { measured }}, y_{i-\text { measured }}, z_{i-\text { measured }}\right)$ (called direct method). The second one is based on Kriging described as follow. The height value of $\mathrm{Z}$ has spatial correlation with the positions $\mathrm{X}$ and $\mathrm{Y}$. Thus, the estimation of the value $\mathrm{Z}$ is calculated by Kriging Method $z_{0}^{*}=f\left(x_{i-\text { measured }}, y_{i-\text { measured }}\right)$. The calibration is obtained by the difference between the estimated value $z_{0}$ and the projection point on the fitting plane $\Delta z_{0}=z_{0}^{*}$ (point predicted $)-z$ (point projected on the fitting plane $)$. For the diagonal plane, measurement error along $\mathrm{Y}$ axis is calculated in the same way.

\section{[Insert Fig. 13 here]}

Calibrations in both areas are estimated by the Kriging method, the calibrations $\Delta z_{0}$ in the cubic space and on the fitting plane are shown in Fig. 14. The left image shows the estimated calibration, and the right image is the variance of estimation. 


\section{[Insert Fig. 14 here]}

The distribution of the estimated calibration depends greatly on the distribution of the measurement error. If the point is closer to the expected measured points, the variance of the estimate is lower. The variance of the estimation in the cubic space is symmetrical along the gauge plane and lower around the gauge plane.

It is important to choose the appropriate Kriging method including correlation model and regression model corresponding to the sampling strategy in order to reduce the estimation variance. Table 3 shows that the results of estimation and variance in process of calibration. According to Table 3, when the exponential model is chosen as the correlation model and Ordinary Kriging is chosen as the regression model in Fig. 14(a) and Fig. 14 (b) , and Universal Kriging is chosen in Fig. 14 (c) respectively, the estimation variances $\sigma_{\hat{Z}}^{2}\left(s_{0}\right)$ are minimized.

\section{[Insert Tab. 3 here]}

\subsubsection{Sphere case}

Two experiments were conducted to provide calibrations in various areas: in the cubic space and on the gauge sphere shown in Fig. 15. The estimation points on the spherical datum surface are shown in Fig. 16, and results of calibration estimation and estimation error variance on the spherical datum surface are shown in Fig. 17. The calibration of GOM sensor can be achieved at any sites of the full-field area of measurement by the proposed Kriging method. For a new measurement mission, the calibration of the measurement system can be automatically compensated to the results of the measures.

\section{[Insert Fig. 15 here]}

[Insert Fig. 16 here]

[Insert Fig. 17 here] 


\subsection{Sensitivity analysis of different numbers of points to construct meta-model}

The cross-validation method not only helps to choose the appropriate Kriging method to estimate the calibration of the GOM sensor, but also assists to decide the number of measurement error applied to construct meta-model of calibration. The sensitivity analysis on different initial number of points to construct meta-model is conducted according to the process of cross-validation in Fig. 4, and the main steps are described as follows.

1) Generate the measurement error $\Delta z_{i}$ by the $\mathrm{N}$ measured points $(\mathrm{N}=33678)$.

2) Randomly select $M=10,50,100,300,500$ points from the $N$ measured points for the estimation of calibration and temporarily eliminate the measurement error of the remaining points $(\mathrm{N}-\mathrm{M})$.

3) Calculate the estimation and Kriging variance associated in the remaining points.

4) Repeat 50 times steps (2) to (3) for each M points.

5) Calculate the cross-validation criterion

6) Build the box plot (box-whisker plot) of cross-validation criterion for evaluation.

Fig. 18 shows the results of different cross-validation criterion ME, MSE, RMSE, AKSE, MSPE and RMSPE with different numbers of measured points for the creation of meta-model Kriging.

\section{[Insert Fig. 18 here]}

According to Fig. 18, some conclusions can be obtained:

1) The average estimation errors (ME) and standard errors (MSE) is closer to 0 with the increasing number.

2) The variance of estimation errors (MSE, RMSE) is lower with greater number of points. This criterion reflects the robustness of the estimator and information on the accuracy of the estimation.

3) The values of MSPE with different numbers of points converge to 0. But RMSPE has a fluctuation with the increasing number of points, and at last converges to the specific value, 1. 
Thus, the accuracy of the estimation of calibrations is increased by the number of measured points for the creation of meta-model. But the accuracy is priced by measurement cost and the calculation time. Generally, the more number of points used to estimate the calibration by Kriging method, the higher accuracy of estimation is achieved. So it is important to choose the appropriate number of points for the meta-model creation. The best indication is the variability RMSPE.

- $\quad$ RMSPE $=1$ indicates that the prediction of the calibration is correct and effective.

- RMSPE $>1$ indicates that the prediction is overestimated, which means to reduce the measurement information.

- $\quad$ RMSPE $<1$ indicates that the prediction is underestimated, which means to increase the measurement information.

The variability of RMSPE by Ordinary Kriging and by Universal Kriging are shown in Fig. 19. For Ordinary Kriging, 50 or 100 measured points lead to a less than 1 RMSPE, but for 500 points it is greater than 1 . This indicates that 500 measured points are sufficient for the prediction of calibrations. If more points are used for the calculation, the variability of the prediction will be overestimated. For Universal Kriging, 500 measured points are not enough to perform the calibration. For an industrial application, a reference threshold of RMSPE could be defined to optimize the needed number of measured points.

\section{[Insert Fig. 19 here]}

\subsection{Spatial correlation analysis for measurement uncertainty}

A convenient choice for the correlation function is within the power exponential model and spherical model. Spatial correlation functions are $\boldsymbol{d}=\left\|\boldsymbol{s}_{i}-\boldsymbol{s}_{j}\right\|$

- $\quad$ Exponential model: $R_{j}\left(\theta, d_{j}\right)=\exp \left(-\theta_{j}\left|d_{j}\right|\right)$

- Gaussian model: $R_{j}\left(\theta, d_{j}\right)=\exp \left(-\theta_{j} d_{j}{ }^{2}\right)$

- Spherical model: $R_{j}\left(\theta, d_{j}\right)=1-1.5 \xi_{j}+0.5 \xi_{j}{ }^{3}, \xi_{j}=\min \left\{1, \theta_{j}\left|d_{j}\right|\right\}$

Fig. 20 shows the spatial correlation influenced by distance and $\theta$. From Fig. 20, it is 
observed that the correlation decreases with $\boldsymbol{d}$ and a larger value for $\theta$ leads to a faster decrease.

\section{[Insert Fig. 20 here]}

\section{Conclusion}

In this paper, a novel Kriging models-based calibration and uncertainty estimation method is developed to improve the measurement accuracy of non-contact 3D CMSs. This calibration procedure has been tested on a measurement system with the GOM sensor, which served as a tool of measurement after calibration. The results of calibrations are used to compensate and correct the measurement system to perform a new measure. The cross-validation technique is applied to select the best Kriging model and decide the initial measured points to create the meta-model. The accuracy of the results and the effective of the calibration estimation impact on the measurement time and on the computation time. The results show that fitted calibration surface obtained through Kriging-based method can provide accurate estimation of the calibration for the new measurement. As shown in the figure 17 , the accuracy of the results with the kriging compensation is 10 times the accuracy of the results without the kriging compensation. 


\section{References}

[1] P. T. Kolen, "Self-calibration/compensation technique for microcontroller-based sensor arrays," IEEE Transaction on Instrumentation and Measurement, vol. 43, no. 9, pp. 620-623, Aug. 1994.

[2] H. Bacakoglu, and M. S. Kamel, "A three-step camera calibration method," IEEE Transaction on Instrumentation and Measurement, vol. 46, no. 5, pp. 1165-1172, Oct. 1997.

[3] G. Sansoni, M. Carocci and R. Rodella, "Calibration and performance evaluation of a 3-D imaging sensor based on the projection of structured light," IEEE Transaction on Instrumentation and Measurement, vol. 49, no. 3, pp. 628-636, Jun. 2000.

[4] T. S. Shen, and C. H. Meng, "Automatic camera calibration for a multiple-sensor integrated coordinate measurement system," IEEE Transaction on Robotics and Automation, vol. 17, no. 4, pp. 502-507, Aug. 2001.

[5] S. Du, C. Liu and L. Xi, "A selective multiclass support vector machine ensemble classifier for engineering surface classification using high definition metrology," ASME Transaction on Journal of Manufacturing Science and Engineering, vol. 137, 011003-1-15, 2015.

[6] S. Du, D. Huang, and H. Wang, "An adaptive support vector machine-based workpiece surface classification system using high definition metrology," IEEE Transaction on Instrumentation and Measurement, vol. 64, no. 10, pp. 2590-2604, 2015.

[7] H. Nguyen, H. Wang, and S. J. Hu, "Characterization of Cutting Force Induced Surface Shape Variation in Face Milling Using High-Definition Metrology," ASME Transaction on Journal of Manufacturing Science and Engineering, vol.135, no. 4, pp. 041014-1-12, 2013.

[8] H. Nguyen, H. Wang, B.L. Tai, J. Ren, S.J. Hu and A. Shih, "High-Definition Metrology Enabled Surface Variation Control by Cutting Load Balancing," ASME Transaction on Journal of Manufacturing Science and Engineering, vol. 138, no. 2, pp. 021010-1-11, 2016.

[9] M. Wang, L. Xi, and S. Du, "3D Surface form Error Evaluation using High Definition Metrology," Precision Engineering, vol. 38, no. 1, pp. 230-236, 2014.

[10] S. Du, C. Liu, and D. Huang, "A Shearlet-Based Separation Method of 3D Engineering 
Surface Using High Definition Metrology,” Precision Engineering, vol. 40, pp. 55-73, 2015.

[11] M. Wang, Y. Shao, S. Du and L. Xi, "A diffusion filter for discontinuous surface measured by high definition metrology," International Journal of Precision Engineering and Manufacturing, vol. 16, no. 10, pp. 2057-2062, 2015.

[12] M. Wang, T. Ken, S. Du, and L. Xi, "Tool Wear Monitoring of Wiper Inserts in Multi-insert Face Milling Using 3D Surface Form Indicators," ASME Transaction on Journal of Manufacturing Science and Engineering, vol. 137, no. 3, pp. 031006-1-7, 2015.

[13] B. Pan, L. Yu, D. Wu, and L. Tang, "Systematic errors in two-dimensional digital image correlation due to lens distortion," Optics and Lasers in Engineering, vol. 51, no. 2, pp. 140147, 2013.

[14] S.C. Cui, Z. Xiao, "A generalized reference-plane-based calibration method in optical triangular profilometry," Optics Express, vol. 17, no. 23, pp.20735-20746, 2009.

[15] B. Pan, L. Yu, D. Wu, and L. Tang, "Systematic errors in two-dimensional digital image correlation due to lens distortion," Optics and Lasers in Engineering, vol. 51, no. 2, pp.140-7, 2013.

[16] P. S. Huang and J. Ni, "On-line error compensation of coordinate measuring machines," International Journal of Machine Tools and Manufacture. vol. 35, no. 5, pp. 725-738, 1995.

[17] O. Satoa, S. Osawaa, Y. Kondob, M. Komoric, and T. Takatsujia, "Calibration and uncertainty evaluation of single pitch deviation by multiple-measurement technique," Precision Engineering, vol. 34, no. 1, pp. 156-163, 2010.

[18] E.M. Barini, G. Tosello, and L.D. Chiffre. "Uncertainty analysis of point-by-point sampling complex surfaces using touch probe CMMs_ DOE for complex surfaces verification with CMM,” Precision Engineering, vol. 34, no. 1, pp. 16-21, 2010.

[19] C. Hong, and S. Ibaraki. "Non-contact R-test with laser displacement sensors for error calibration of five-axis machine tools," Precision Engineering, vol. 40, no. 1, pp. 159-171, 2013.

[20] M. Kajima, and K. Minoshima1. "Calibration of linear encoders with sub-nanometer uncertainty using an optical-zooming laser interferometer," Precision Engineering, vol. 38, no. 
4, pp. 769-774, 2014.

[21] C.J. Evans, "Precision Engineering: An Evolutionary View," Cranfield, U. K.: Cranfield University Press, 1989.

[22] Y.D. Chen and J. Ni, "Dynamic calibration and compensation of a 3D laser radar scanning system," IEEE Transaction on Robotics and Automation, vol. 9, no. 3, pp. 318-323, Jun. 1993.

[23] J. Caja, E. Gómez, and P. Maresca. "Optical measuring equipments. Part I_ Calibration model and uncertainty estimation," Precision Engineering, vol. 40, pp. 298-304, 2015.

[24] Y. Shen, and S. Moon, "Error compensation of coordinate measurements in computer-Integrated manufacturing using neural networks," Journal of Material Processing Technology, vol. 61, no. 1, pp. 12-17, 1996.

[25] Q. Yang, C. Butler, and P. Baird, "Error compensation of touch trigger probes," Measurement, vol. 18, no. 1, pp. 47-57, 1996.

[26] C. Dong, C. Zhang, B. Wang, and G. Zhang, "Prediction and compensation of dynamic errors for coordinate measuring machines," ASME Transaction on Journal of Manufacturing Science and Engineering, vol. 124, no. 3, pp. 509-514, 2002.

[27] K.K. Tan, S.N. Huang, and H.L. Seet, "Geometrical error compensation of precision motion systems using radial basis function," IEEE Transaction on Instrumentation and Measurement, vol. 49, no. 5, pp. 984-991, Oct. 2000.

[28] K.K. Tan, S.N. Huang, and T.H. Lee, "Dynamic S-function for geometrical error compensation based on neural network approximations," Measurement, vol. 34, no. 2, pp. 143-156, Sep. 2003.

[29] N. A. Barakat, M. A. Elbestawi, and A. D. Spence, "Kinematic and geometric error compensation of a coordinate measuring machine," International Journal of Machine Tools \& Manufacture, vol. 40, no. 6, pp. 833-850, 2000.

[30] X. H. Li, B. Chen, and Z. R. Qiu, "The calibration and error compensation techniques for an articulated arm CMM with two parallel rotational axes," Measurement, vol. 46, no. 1, pp. 603-609, 2013. 
[31] Y. Bai and H. Zhuang, "On the comparison of bilinear, cubic spline, and fuzzy interpolation techniques for robotic position measurements," IEEE Transaction on Instrumentation and Measurement, vol. 54, no. 6, pp. 2281-2288, Dec. 2005.

[32] C. Teo, K. Tan, and S. Lim, "Dynamic geometric compensation for gantry stage using iterative learning control," International Journal of Machine Tools \& Manufacture, vol. 57, no. 2, pp. 413-419, 2008.

[33] S. L. Yang, and K. J. Xu, "Numerical Derivation-based serial iterative dynamic decoupling-compensation method for multiaxis force sensors," IEEE Transactions on Instrumentation and Measurement, vol. 63, no.12, pp. 2950-2962, Dec. 2014.

[34] J. Wei, and Y. Chen, "The geometric dynamic errors of CMMs in fast scanning-probing," Measurement, vol. 44, no. 3, pp. 511-517, 2011.

[35] X. Wen, Y. Zhao, D. Wang, and J. Pan. "Adaptive Monte Carlo and GUM methods for the evaluation of measurement uncertainty of cylindricity error," Precision Engineering, vol. 37, no. 4, pp. 856-864, 2013.

[36] H. Feng, Y. Liu, and F. Xi, "Analysis of digitizing errors of a laser scanning system," Precision Engineering, vol. 25, no. 3, pp. 185-191, 2001.

[37] A. Isheil, J. P. Gonnet, D. Joannic, and J. F. Fontaine, "Systematic error correction of a 3D laser scanning measurement device," Optics \& Lasers in Engineering, vol. 49, no. 1, pp. 16-24, 2011.

[38] R. P. Johnson, and Q. Yang, "Dynamic error characteristics of touch trigger probes fitted to coordinate measuring machines," IEEE Transactions on Instrumentation and Measurement, vol. 47, no. 5, pp. 1168-1172, Oct. 1998.

[39] P. H. Pereira, and R. J. Hocken, "Characterization and compensation of dynamic errors of a scanning coordinate measuring machine," Precision Engineering, vol. 31, no. 1, pp. 22-32, 2007.

[40] H. Schwenke, W. Knapp, H. Haitjema, A. Weckenmann, R. Schmitt, and F. Delbressine, "Geometric error measurement and compensation of machines-an update," CIRP Annals-Manufacturing Technology, vol. 57, no. 2, pp. 660-675, 2008. 
[41] G. X. Zhang, H. Y. Zhang, J. B. Guo, X. H. Li, Z. R. Qiu, and S. G. Liu, "Error compensation of cylindrical coordinate measuring machines," CIRP Annals-Manufacturing Technology, vol. 59, no. 1, pp. 501-504, 2010.

[42] S. Suriano, H. Wang, C. Shao, S. J. Hu, and P. Sekhar, "Progressive measurement and monitoring for multi-resolution data in surface manufacturing considering spatial and cross correlations," IIE Transaction, vol. 47, no. 10, pp. 1033-1052, 2015.

[43] M. Poniatowska, "Research on spatial interrelations of geometric deviations determined coordinate measurements of free-form surfaces," Metrology \& Measurement systems, vol. 16, no. 3, pp. 501-510, 2009.

[44] X. Chen, B.E. Ankenman, and B.L. Nelson, "Enhancing stochastic Kriging metamodels with gradient estimators," Operations Research, 61, 512-528, 2013.

[45] J.P.C. Kleijnen, "Kriging metamodeling in simulation: A review," European Journal of Operational Research, vol. 192, pp. 707-716, 2009.

[46] S. Du and L. Fei, "Co-Kriging method for form error estimation incorporating condition variable measurements," ASME Transaction on Journal of Manufacturing Science and Engineering. vol. 138, pp. 041003-1-16, 2016.

[47] R. Ascione, G. Moroni, S. Petro, and D. Romano, "Adaptive inspection in coordinate metrology based on kriging models," Precision Engineering, vol. 37, no. 1, pp. 44-60, 2013. [48] A. Dumas, B. Echard, N. Gayton, O. Rochat, J. Y. Dantan, and S. V. D. Veen, “AK-ILS: an active learning method based on Kriging for the inspection of large surfaces," Precision Engineering, vol. 37, no. 1, pp. 1-9, 2013.

[49] Y. Chen, J. Gao, H. Deng, D. Zheng, X. Chen, and R. Kelly, "Spatial statistical analysis and compensation of machining errors for complex surfaces," Precision Engineering, vol. 37, no. 1, pp. 203-212, 2013.

[50] K. Fan, J. Yang, and L. Yang, "Unified error model based spatial error compensation for four types of CNC machining center: Part II-unified model based spatial error compensation,” Mechanical Systems \& Signal Processing, vol. 49, no. 1-2, pp. 63-76, 2014.

[51] K. Fan, J. Yang, and L. Yang, "Unified error model based spatial error compensation for 
four types of CNC machining center: Part I-Singular function based unified error model," Mechanical Systems \& Signal Processing, vol. 60, no. 1-2, pp. 656-667, 2015. 


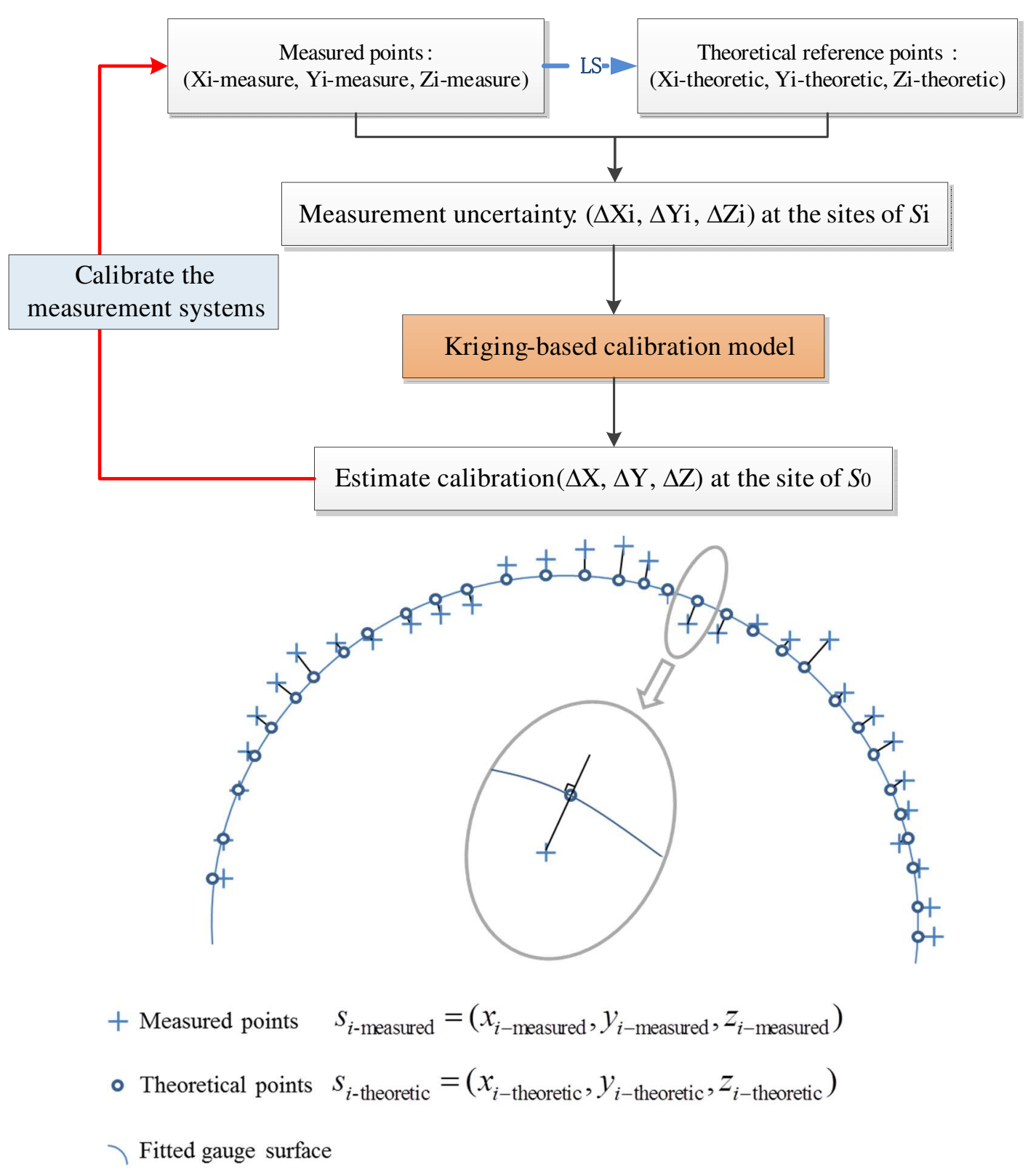

Fig. 1. The calibration procedure 


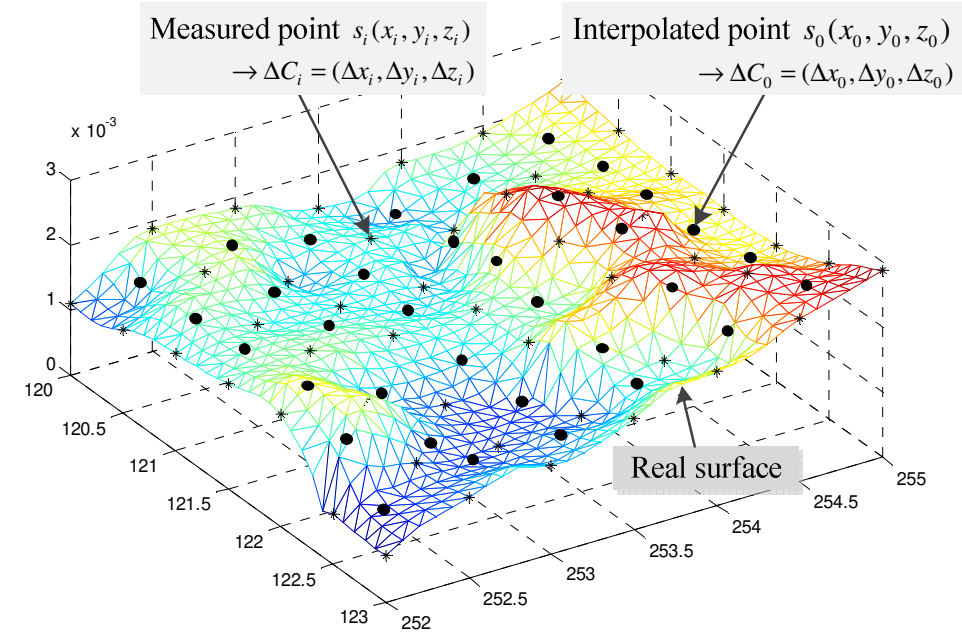

Fig. 2. Measured point and interpolated point

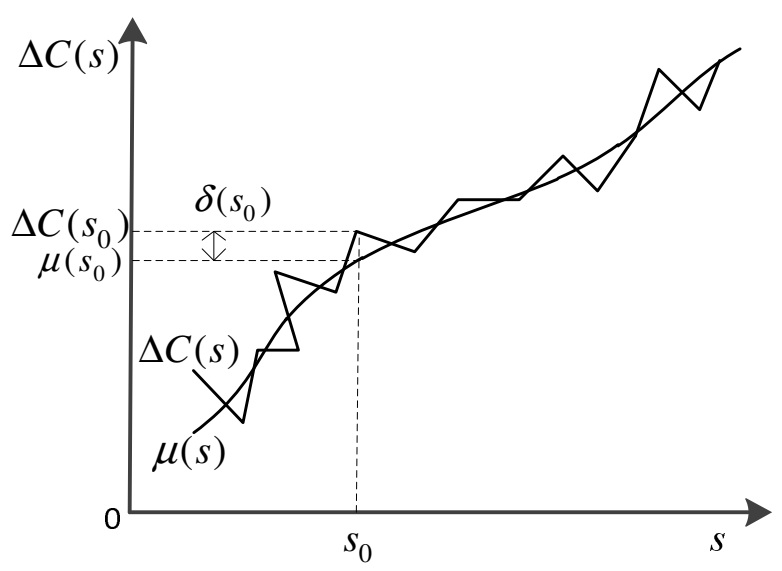

Fig. 3. Measurement error components 
Generate measurement error with three dimensions $\Delta \mathrm{C}$ by $\mathbf{N}$ measured points and those projected on the fitting plan

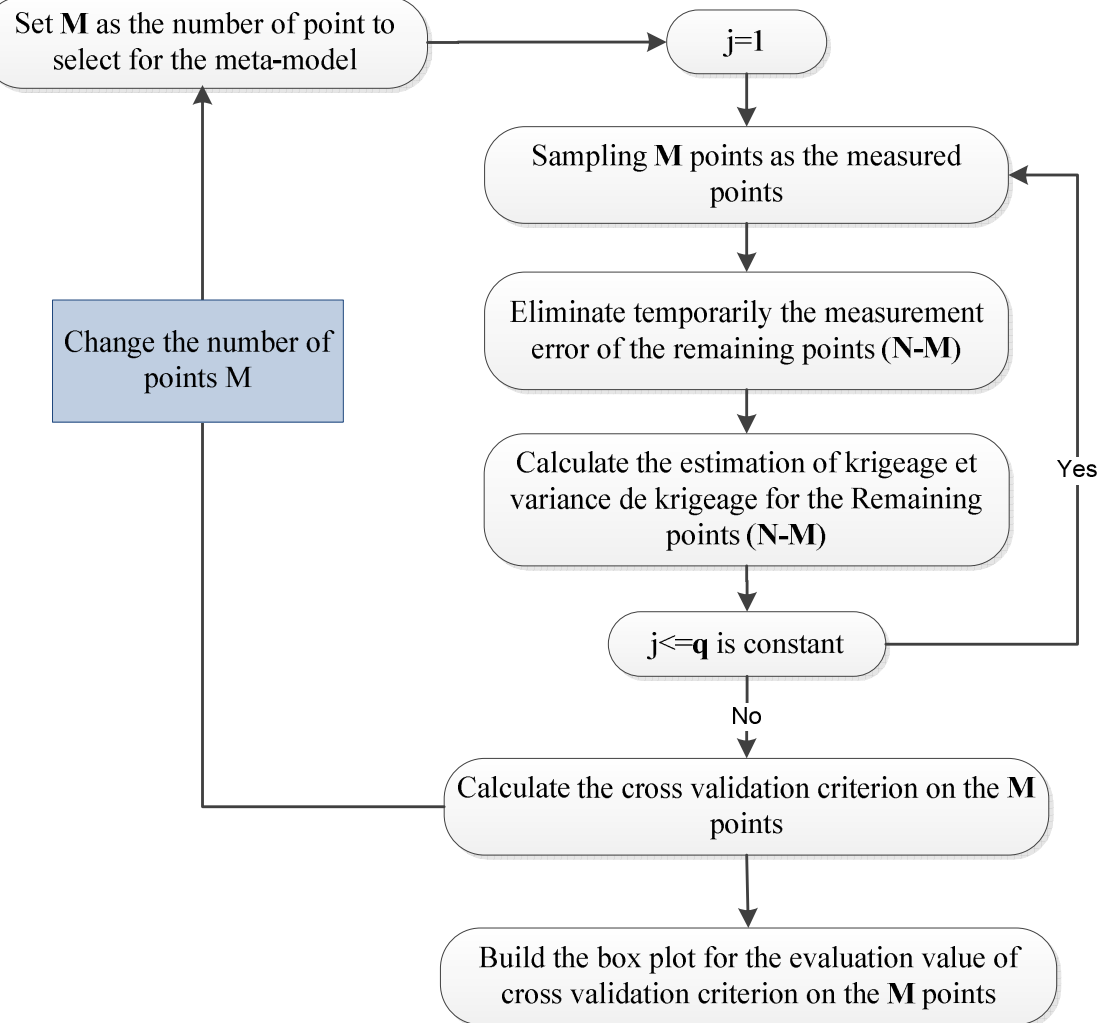

Fig. 4. Procedure of cross validation 
Table 1 The fitting planes by LS method

\begin{tabular}{|c|c|c|}
\hline Plane & Number of points & Gauge plane \\
\hline Lower plane & 33678 & $\mathrm{z}=-122.9953+0.00010 * \mathrm{x}-0.0516 * \mathrm{y}$ \\
\hline Middle plane & 41051 & $\mathrm{z}=5.5013+0.001953 * \mathrm{x}-0.05085 * \mathrm{y}$ \\
\hline Upper plane & 31894 & $\mathrm{z}=78.7114+0.003069 * \mathrm{x}-0.04998 * \mathrm{y}$ \\
\hline Diagonal plane & 57649 & $\mathrm{z}=20.2790-0.0125 * \mathrm{x}-0.9290 * \mathrm{y}$ \\
\hline
\end{tabular}

Table 2. The fitting sphere by LS method

\begin{tabular}{|c|c|c|c|}
\hline & $\mathrm{X}$ & $\mathrm{Y}$ & $\mathrm{Z}$ \\
\hline Gauge center of sphere & -85.2707 & 65.6985 & -55.4355 \\
\hline Measured radius & \multicolumn{3}{|c}{$15.0088 \mathrm{~mm}$} \\
\hline Gauge radius & \multicolumn{3}{|c}{$15.0004 \mathrm{~mm}$} \\
\hline
\end{tabular}

Table 3. Results of estimation and variance in process of calibration

\begin{tabular}{|c|c|c|c|c|c|c|}
\hline \multirow{2}{*}{} & \multicolumn{2}{|c|}{ In the cubic space } & \multicolumn{2}{c|}{$\begin{array}{c}\text { On the fitting plane by } \\
\text { Direct method }\end{array}$} & $\begin{array}{c}\text { On the fitting plane by } \\
\text { Kriging method }\end{array}$ \\
\cline { 2 - 7 } & Estimation & Variance & Estimation & Variance & Estimation & Variance \\
\hline Mean & & & $2.08 \mathrm{E}-04$ & $5.50 \mathrm{E}-05$ & $2.30 \mathrm{E}-04$ & $4.99 \mathrm{E}-05$ \\
\hline Min & $-7.61 \mathrm{E}-02$ & $2.40 \mathrm{E}-05$ & $-7.94 \mathrm{E}-02$ & $4.88 \mathrm{E}-06$ & $-8.31 \mathrm{E}-02$ & $1.39 \mathrm{E}-06$ \\
\hline Max & $9.03 \mathrm{E}-02$ & $7.10 \mathrm{E}-04$ & $9.92 \mathrm{E}-02$ & $1.90 \mathrm{E}-04$ & $1.08 \mathrm{E}-01$ & $1.71 \mathrm{E}-04$ \\
\hline $\begin{array}{c}\text { Regression } \\
\text { model }\end{array}$ & Ordinary Kriging & Universal Kriging \\
\hline $\begin{array}{c}\text { Correlation } \\
\text { model }\end{array}$ & \multicolumn{7}{c|}{ Exponential model } \\
\hline
\end{tabular}

